Research Article

\title{
Solving a Real-World Urban Postal Service System Redesign Problem
}

\author{
Hao Yu $\mathbb{D}^{1}{ }^{1}$ Xu Sun, ${ }^{1}$ Wei Deng Solvang, ${ }^{1}$ and Gilbert Laporte ${ }^{2,3}$ \\ ${ }^{1}$ Department of Industrial Engineering, UiT The Arctic University of Norway, Lodve Langsgate 2, Narvik 8514, Norway \\ ${ }^{2}$ Department of Decision Sciences, HEC Montréal, 3000 Chemin de la Côte-Sainte-Catherine, Montréal H3T 2A7, Canada \\ ${ }^{3}$ School of Management, University of Bath, Bath, UK
}

Correspondence should be addressed to Hao Yu; hao.yu@uit.no

Received 22 April 2021; Revised 16 May 2021; Accepted 20 May 2021; Published 28 May 2021

Academic Editor: Tingsong Wang

Copyright (@ 2021 Hao Yu et al. This is an open access article distributed under the Creative Commons Attribution License, which permits unrestricted use, distribution, and reproduction in any medium, provided the original work is properly cited.

\begin{abstract}
Due to recent technological advancements, more diversified customer demand, and increasingly harder competition, traditional postal service systems have experienced significant changes all over the world. In Norway, through a strategic reform called postin-shop, undertaken in 2013, most postal services are now provided at postal service counters located in retailer stores in order to improve accessibility, operational efficiency, and cost-effectiveness. This has led to a complex decision-making problem for the redesign of urban postal service networks across the country. In this paper, a two-stage method is proposed to solve a real-world urban postal service network redesign problem. First, two location models are employed to determine the optimal locations of postal service counters. In the second stage, a simulation model is built to evaluate the urban postal service system with different location and demand allocation plans under a realistic and stochastic environment. Among other insights, our results show that the proposed two-stage method can be used to effectively improve the accessibility of postal service networks by making optimal location-allocation decisions.
\end{abstract}

\section{Introduction}

In recent years, with the rapid development and increasing use of computer technologies, the traditional postal service system has experienced significant changes in order to better satisfy customer demand and to survive in a more competitive market $[1,2]$. The wide adoption of digitalization and information and communication technology (ICT) across businesses, public sectors, and individuals has led to two major challenges in the postal service system. On the one hand, customer demand for traditional letter delivery services has declined drastically due to the extensive use of electronic platforms, e.g., e-mail and digital mailboxes $[1,3]$. However, on the other hand, the technological development has also altered customer behavior, and the customer demand fulfilled by online channels has increased unprecedentedly [4]. At the same time, the growth of online shopping has generated a significant increase in customer demand for parcel delivery [5]. Furthermore, due to the increased labor cost and the emergence of international competitors for parcel delivery, e.g., DHL, UPS, and FedEx, the traditional postal service system faces a much harder competition [6]. Hence, worldwide postal service providers need to shift their focus and replan their postal service systems to improve operational efficiency and accessibility.

In Norway, an ambitious strategic reform and structural change of the urban postal service network, named post-inshop, was launched by Posten Norge in 2013. The scheme has been implemented across the whole country with the aim of converting the traditional centrally located post offices into smaller postal service counters at local supermarkets and grocery stores [3]. Through a partnership with retailers, Posten Norge can not only reduce the cost of opening and operating large dedicated post offices in city centers but also can improve the accessibility of postal service by locating a larger number of postal service counters that are closer to customer demand. Meanwhile, the utilization of space and personnel at local supermarkets and grocery stores can also be improved. 
To implement this scheme optimally, an analysis of the urban postal service network redesign problem is essential. The problem can be modeled as a service facility location problem, which aims at selecting the optimal facility locations to provide services and determining the allocation of customer demand. Facility location is one of the most crucial decisions in strategic management throughout a wide range of businesses and public sectors [7, 8]. Mathematical models, as well as other qualitative and quantitative approaches, such as analytical hierarchy process (AHP) $[9,10]$, geographical information systems (GIS) [11, 12], and simulation [13], have been investigated and applied to the optimization of facility locations with respect to different criteria.

In service facility location problems, one widely formulated objective is to maximize the accessibility of a service network to targeted customer demand [14]. Accessibility can be measured either by the coverage of customer segments $[15]$ or by the distance or travel time $[14,16]$. To improve the accessibility of an urban postal service system, we proposed a two-stage optimization-simulation approach, where two classical location models were first employed to choose the best locations of postal service counters, and a simulation model was then applied to evaluate different facility location and demand allocation strategies. One reason for applying simulation after the optimization phase is that several reallife features, namely, stochasticity, cannot be readily incorporated into the mathematical models. In addition, the output of the optimization models depends on the aggregation levels used for the inputs.

This paper aims at effectively using the strengths of both optimization and simulation to solve a real-world urban postal service network redesign problem. Even though the problem is motivated and derived from a specific problem in Norway, the methodological framework can be adopted in a wide range of applications. The remainder of this paper is organized as follows. With a focus on practical applications, Section 2 presents an extensive literature review on the location models and on simulation for network optimization problems. This is followed by the problem description in Section 3. Section 4 introduces the methodology. Section 5 is devoted to data generation, results, and discussions. Conclusions follow in Section 6.

\section{Literature Review}

The redesign of postal networks has been the object of attention in recent years. Mathematical models were developed to analyze cost efficiency [17] and optimize network replanning decisions, i.e., postbox locations $[1,2]$. The urban post office relocation problem was modeled by Šarac et al. [18] who used a set covering location problem (SCLP) to determine the minimum number of permanent post offices required for providing a certain level of service. However, due to the resource limitation in a large number of realworld problems, the urban postal network may not be able to provide a desired level of coverage to all customers. Thus, this paper focuses on improving the accessibility of an urban postal service system with a limited number of postal service counters installed.
The redesign of an urban postal service network belongs to service facility location problems, which have been investigated by a large number of researchers [19]. In this paper, we proposed a two-stage method where service facility location models with budgetary constraints were first used to generate optimal locations. Then, a simulation model was developed and used for performance evaluation. Hence, our literature review focuses on the recent development and applications with respect to the aforementioned methods.

2.1. Maximal Covering Location Problem. The maximal covering location problem (MCLP) was put forward by Church and ReVelle [20]. Over the years, the MCLP has become one of the most extensively investigated location models due to its applicability to a wide range of practical problems [21]. In the MCLP, the distance or the travel time between customers and facilities is the measurement of the satisfaction of customer demand [20]. The MCLP aims at maximizing the coverage of weighted customer demand with limited facilities [22].

Recent research has focused on the development of improved models and computational algorithms for the MCLP [23]. Seyhan et al. [24] proposed a new model and heuristic for a MCLP under competition between different players. Janković et al. [25] improved the formulation and solution approach of an uncapacitated single or multiple allocation $p$-hub MCLP. Combining both exact and metaheuristic methods, Pereira et al. [26] investigated an efficient solution approach for a probabilistic maximal covering location-allocation problem. Cordeau et al. [27] developed an enhanced Benders decomposition approach to solve large maximal covering models.

Real-world applications of the MCLP have also been documented. The improvement of the accessibility of healthcare facilities with improved MCLPs was studied by Murawski and Church [28]; Verter and Lapierre [29]; and Sorensen and Church [30]. Recently, Frade and Ribeiro [31] investigated the optimal design of an urban bike-sharing system under a budget constraint. Pasandideh et al. [32] proposed a multiobjective hub MCLP to simultaneously maximize both network reliability and commodity flow. Taking into account the uncertainty related to the impact of disasters, Li et al. [33] formulated a cooperative MCLP for the design of a humanitarian relief logistics network. Paul et al. [34] formulated an improved biobjective MCLP for the network redesign of a large-scale emergency response system. The model aims at simultaneously maximizing the demand coverage and minimizing the efforts needed for modifying the existing system.

2.2. $p$-Median Location Problem. The $p$-median problem (PMP) was first formulated by Hakimi $[35,36]$, where it was used to determine the optimal locations of switching centers in a telecommunications network. The PMP aims at minimizing the total travel distance or travel time by opening a fixed number $p$ of service facilities [37]. To better cope with practical problems, several extensions of the classic PMP have been formulated, e.g., the capacitated PMP [38], the 
Hamiltonian PMP [39], the $p$-hub median problem [40], and the competitive PMP [41].

The PMP is NP-hard (see, e.g., [42]), so the computational performance with both improved heuristics and commercial solvers has been tested in recent works [43]. Hale et al. [44] solved a large-scale classic PMP with an improved Lagrangian search approach. Erdoğan et al. [45] and Bektaş et al. [46] tested different exact and approximation methods to solve a Hamiltonian PMP. Stefanello et al. [47] proposed an iterated reduction matheuristic combining both mathematical programming techniques and local search metaheuristics to solve a capacitated PMP. Drezner and Salhi [48] solved a planar PMP using efficient neighborhood reductions combined with metaheuristics. Colmenar et al. [49] put forward an enhanced greedy randomized adaptive search approach for an obnoxious PMP.

Focusing on real-world applications, De Azevedo and Pizzolato [50] investigated an urban real estate location problem in Rio de Janeiro modeled as a PMP. To cope with the rapid increases in demands, Adler et al. [51] used the $p$ hub median model to evaluate expansion alternatives for an existing airline network in Africa. Wheeler [52] improved the location decisions on police patrol areas with a modified PMP so that both the total travel distance and the inequality of call allocation can be reduced. Cintrano et al. [53] applied a neighborhood search algorithm to determine the best locations of public bike stations in Malaga. In addition, comparisons between both the MCLP and the PMP were reported in several practical applications, e.g., healthcare services [54], printer locations in an university campus [55], and public charging stations for the electric vehicle [16].

2.3. Computer-Based Simulation. Real-world decisions are rarely made without uncertainty [56]. To capture the stochasticity of a decision-making problem, one may need to employ several assumptions and stochastic parameters, which may either reduce the accuracy of the analysis [30] or increase the computational complexity. The rapid development of computer-based simulation has provided opportunities to solve this problem and to explore the system's performance at a more detailed level [57]. Recently, the use of simulation has become an attractive tool to evaluate a model's output in several problems, e.g., factory flow optimization [58], production planning [57], service accessibility [59], and intermodal transportation under uncertainty [60].

Even though computer-based simulation has already been used in a wide variety of industries and in the public sector [61-63], its application in facility location problems has been less common until recently. Considering the flow of customer demands and demographical data, Rouzafzoon and Helo [64] and Helo et al. [65] investigated agent-based simulation modeling to compare different network configurations of service supply chains in Southern Finland. Li et al. [66] simulated a facility fortification problem in order to mitigate the supply chain risk caused by both natural and human-related disasters. Kim et al. [67] tackled the network design problem of a biomass supply chain by using a twophase simulation method. Elia et al. [68] developed a simulation-based framework to evaluate both economic and environmental performances of a reverse logistics system.

2.4. Summary and Scientific Contributions. In strategic location problems, the idea of using the hybrid optimizationsimulation method originated from the mid-1990s (see, e.g., [69]) and was mostly used in emergency medical service (EMS) location problems, where the vehicle busyness and the rate of call response were analyzed with discrete-event simulation [30,70]. However, in service network design problems, the potential of combining both optimization and simulation has not been fully exploited. For this reason, we propose a two-stage optimization-simulation method in this paper to solve a real-world urban postal service network redesign problem, where the focus is accessibility. In the first stage, the MCLP and the PMP are used to select the optimal facility locations. In the second stage, a simulation model is built with AnyLogic to evaluate the location decisions with respect to different demand allocation strategies.

Our scientific contributions are summarized as follows:

(1) We develop a two-stage optimization-simulation method to improve the accessibility of an urban service system with continuous demand distribution

(2) We show the applicability and effectiveness of the proposed method to a real-world urban postal service system redesign problem

(3) With the simulation tool, the network accessibility is evaluated under the stochasticity of time, location, and demand volume

\section{Problem Description}

3.1. Background. The post-in-shop scheme aims to provide postal services in retail outlets where a postal service counter and a storage space for parcels and letters will be installed. In this paper, the problem under investigation is inspired by a real-world case of the post-in-shop relocation problem in Narvik, which is a small but strategically important town in Northern Norway. With one of the largest ice-free deep-water ports inside the Arctic Circle and the world's northernmost railway connection, Narvik is an important transportation hub in the Arctic region, especially for the shipment of highquality iron ore mined in northern Sweden.

The original post office in Narvik was located at a large shopping mall in the city center, but it was closed in 2013 with the implementation of the post-in-shop scheme. Instead of the original large and centrally located post office in Narvik, two postal service counters were relocated at two supermarkets, Coop Extra Bolaget and SPAR Finnbekken, as shown in Figures 1 and 2. The customers were assigned to different postal service counters based only on their postcodes (the customers with postcode 8514 were assigned to SPAR Finnbekken, and those with postcode 8517 were assigned to Coop Extra Bolaget). The relocation of post offices expands the urban postal service network by opening more facilities closer to the customers. However, many 


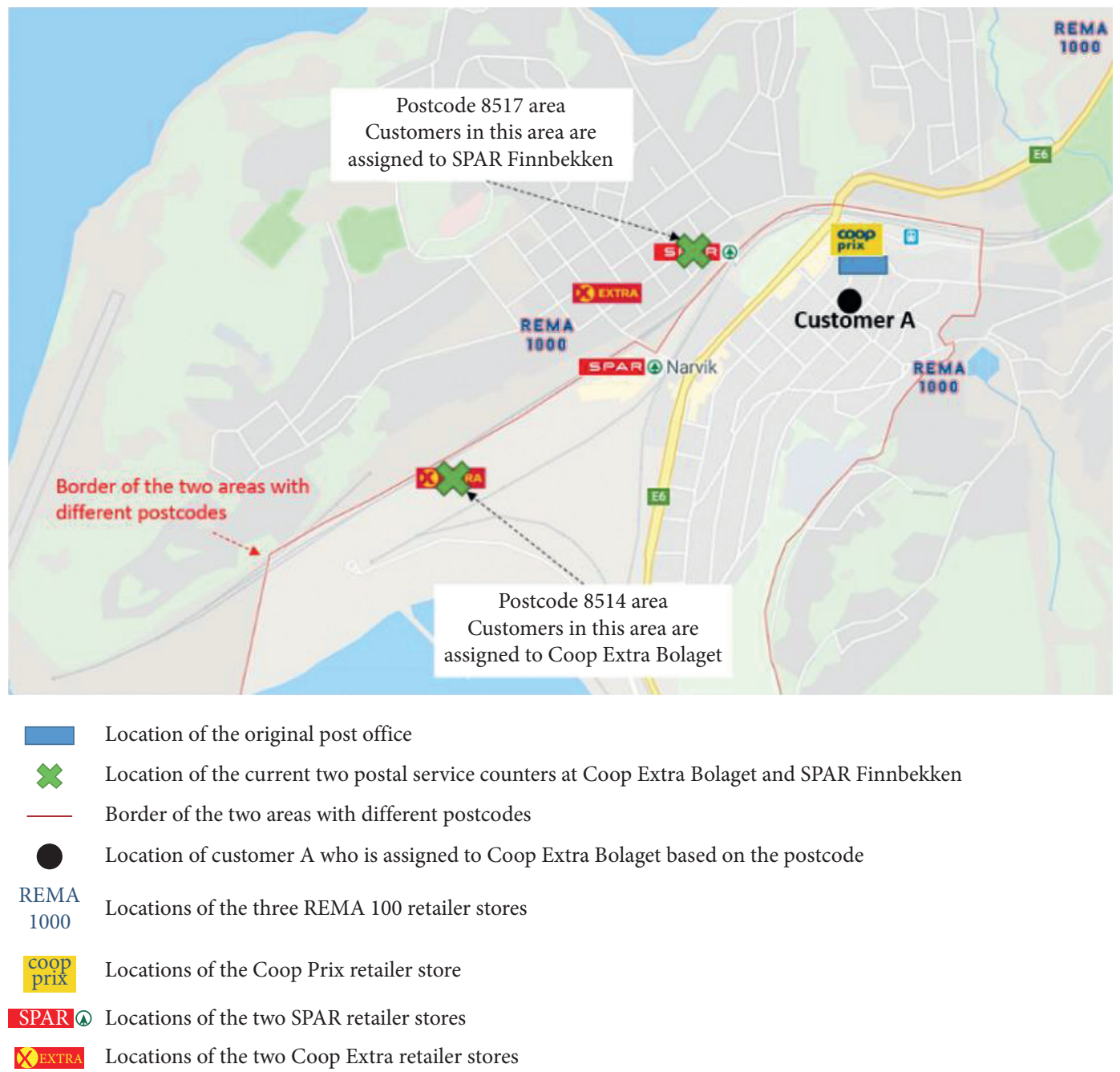

FIGURE 1: Locations of the original post offices, the current postal service counters, and the candidates for postal service counters.

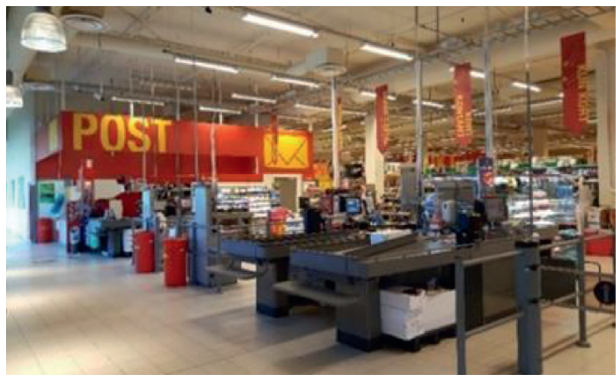

(a)

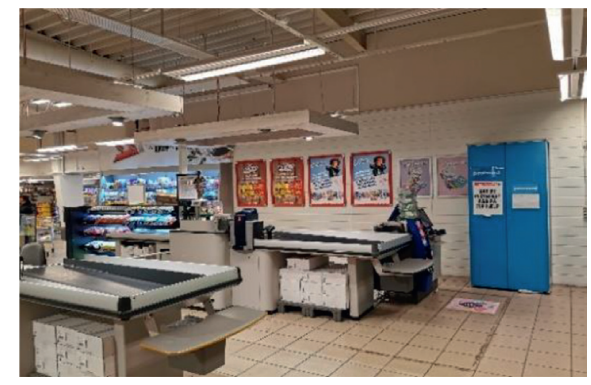

(b)

Figure 2: Post-in-shop at Coop Extra Bolaget and SPAR Finnbekken in Narvik. (a) Coop Extra Bolaget. (b) SPAR Finnbekken.

customers will need to travel a larger distance than before in order to pick up their parcels due to the postcode-based demand allocation. For instance, as shown in Figure 1, customer A located in the postcode 8514 area needs to go to a much further postal service counter (Coop Extra Bolaget) to pick up a parcel instead of going to the closest one (SPAR Finnbekken).
3.2. The Problem. The urban postal service system redesign is, by nature, a service network design problem that determines the locations of service facilities and allocates customers to different locations. In the design of some service systems, e.g., petrol stations and ATM networks, only facility locations need to be determined, and customers are free to choose the facilities for respective services. However, 
for an urban postal service system, customers need to be assigned to specific locations to pick up their parcels. Thus, both facility location and customer allocation decisions are of essential importance to determine the overall performance of this service system in terms of accessibility, convenience, and customer satisfaction. An improperly designed service system, as illustrated in Figure 1, may result in increased travel distance for customers and reduced accessibility. Moreover, this may further increase fuel consumption and hence greenhouse gas emissions. To solve this problem, both facility location and customer allocation decisions need to be optimized.

Designing a service system consists of two-stage decision-making. First, the number and locations of service facilities are selected from a set of candidate points. In an urban postal service system, taking into account the storage space requirement for parcels, the candidate points can only be located at large- and medium-size shops. Figure 1 depicts the candidate locations for potential postal service counters in the urban area of Narvik (three REMA 1000, two SPAR, two Coop Extra, and one Coop Prix). In order to provide a high accessibility to customer demand under budgetary constraints, the determination of the number and locations of service facilities is usually done with the objective either to maximize the demand coverage (the MCLP) or to minimize the total travel distance by customers (the PMP).

Based on the first-stage decisions, the demand allocation can be determined in the second stage, where customers can be either assigned to a specific location or to the nearest facility. In the urban postal system redesign problem, we are interested in the comparison of both postcode-based and distance-based demand allocation strategies, and their implications to the overall system's accessibility are evaluated.

\section{Methodology}

In order to solve the problems of traditional mathematical methods such as demand aggregation and oversimplified assumptions, we proposed a two-stage optimization-simulation method for the service system design problem with special focus on the urban area and tested it with a real-world case study of the urban postal service system redesign in Narvik. We first describe the framework of the two-stage method. Then, the two classical optimization models and the simulation method are briefly introduced.

4.1. Methodological Framework. Figure 3 illustrates a twostage methodological framework for the service system design problem. In the first stage, we apply the MCLP and the PMP to obtain an optimal network configuration with different objectives and requirements. In the second stage, the AnyLogic simulation is used for performance measurement. To set up the simulation model, the GIS information is given based on the optimal locations obtained, and the generation intervals of stochastic parameters are determined in accordance with the data used in the first stage. The agent's behavior is set up based on different demand allocation strategies. It is noted that a stability and quality check is performed so that the proposed method can be used with a high level of confidence in order to solve large problem instances.

In this research, we exploit the strengths of both mathematical optimization and computer-based simulation. The terms of optimization and simulation are used interchangeably in several cases [71]. However, they are two very different techniques that should be properly applied in different problems. Optimization aims, through a model and an algorithm, at searching an optimal objective value within the feasible domain of a problem. It is capable of solving combinatorial optimization problems with a large number of alternatives. However, due to the simplifications made in the models, e.g., demand aggregation, the results obtained may not be sufficiently accurate [30]. Furthermore, the incorporation of input stochasticity into a mathematical model may significantly increase its computational complexity [72].

Simulation, on the contrary, aims at the performance evaluation of a set of alternatives under different conditions [73], which can better reproduce real-world problems with stochastic parameters, real-world GIS, and real planning horizon. Moreover, it is also a powerful tool to create an animation of the physical installations and flows of the system modeled. However, while the primary objective of simulation is for performance evaluation, it can neither solve a complex combinatorial optimization problem nor generate proven optimal decisions when the number of potential alternatives is large. Tables 1 and 2 show the strengths and weaknesses of the two approaches.

4.2. The Location Optimization Models. Both the MCLP and the PMP optimize the network performance under budgetary constraints through installing a limited number of facilities. The sets, parameters, and variables used in the model formulation are first given in Table 3.

The MCLP measures accessibility with the level of demand coverage and aims at establishing a service system to ensure that a maximum number of customers can find a service facility within their preferred distance. In this regard, objective function (1) maximizes the overall weighted customer demand coverage. Constraints (2) guarantee that the demand of customer $i$ is covered only if a service facility is installed within its preferred distance. Constraint (3) specifies the number of facilities to be installed. Constraints (4) define the domain of the variables.

$$
\begin{aligned}
& \text { Maximize } \sum_{i \in I} d_{i} z_{i}, \\
& \text { subject to } \\
& z_{i} \leq \sum_{j \in N_{i}} y_{j}, \quad \forall i \in I, \\
& \sum_{j \in J} y_{j}=p, \\
& z_{i}, y_{j} \in\{0,1\}, \quad \forall i \in I, j \in J .
\end{aligned}
$$




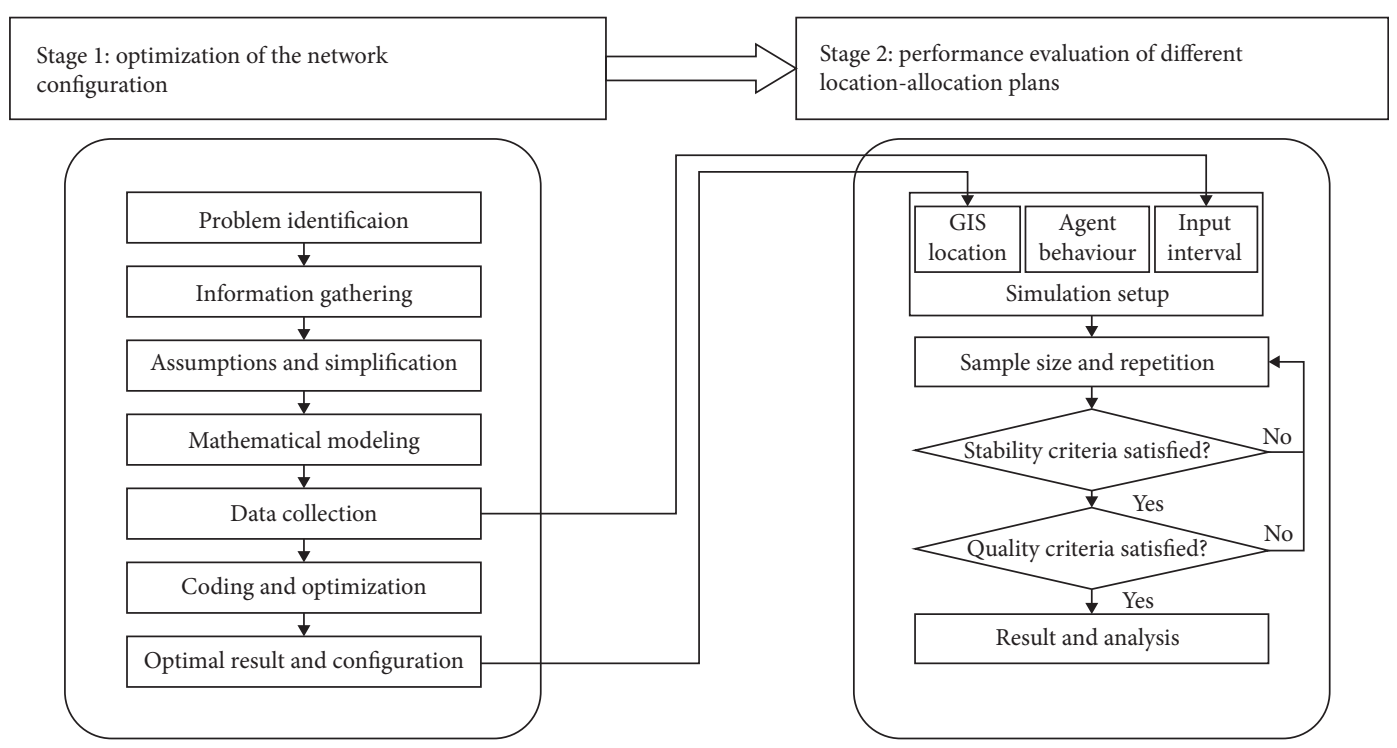

FIGURE 3: Methodological framework.

TABLE 1: Strengths and weaknesses of optimization.

Optimization

Strengths (1) Capability of selecting an optimal solution among a large number of alternatives

(2) Capability of generating high-quality solutions to complex decision-making problems

(1) Inaccurate analytical results due to the simplifications made

Weaknesses

(2) Increased complexity in modeling and computation due to the inclusion of uncertainty

(3) The numerical results may be difficult to be understood by decision makers who do not possess the relevant knowledge

TABLE 2: Strengths and weaknesses of simulation.

Simulation

(1) Capability of evaluating different predetermined alternatives under a realistic environment

Strengths (2) Capability of incorporating the uncertainty, real-world GIS, and time horizon of a decision-making problem

(3) Capability of generating high-quality animation of the physical system

Weaknesses

(1) Ineffectiveness and incapability of selecting an optimal solution among a large number of alternatives

(2) Ineffectiveness and incapability of generating high-quality solutions to complex decision-making problems

TABLE 3: Sets, parameters, and variables.

Sets

I $\quad$ Set of customers, indexed by $i$

$J$ Set of candidate locations for the service facility, indexed by $j$

Parameters

$d_{i}$

$c_{i j}$

$j_{i}=\left\{j \in J: c_{i j} \leq r\right\}$

$p$

Variables

$y_{j}$

$z_{i}$
Demand of customer $i$

Distance between customer $i$ and service facility $j$

The coverage distance of a service facility

The set of candidate locations $j$ of the service facility within the coverage distance of customer $i$ The number of service facilities to be installed

Binary variable: $y_{j}=1$ if a service facility is installed at candidate location $j ; y_{j}=0$, otherwise Binary variable: $z_{i}=1$ if the demand from customer $i$ is covered; $z_{i}=0$, otherwise 
The PMP aims at minimizing the total weighted travel distance by all customers. It not only selects the locations of the service facility but also determines the allocation of customer demands. Hence, an additional variable for demand allocation is defined as follows.

$x_{i j}$ : binary variable: $x_{i j}=1$ if customer $i$ is assigned to service facility $j ; x_{i j}=0$, otherwise.

$$
\begin{gathered}
\text { Minimize } \sum_{i \in I} d_{i} c_{i j} x_{i j}, \\
\text { subject to } \\
\sum_{j \in J} x_{i j}=1, \quad \forall i \in I, \\
x_{i j} \leq y_{j}, \quad \forall i \in I, j \in J, \\
\sum_{j \in J} y_{j}=p, \quad \\
x_{i j}, y_{j} \in\{0,1\}, \quad \forall i \in I, j \in J .
\end{gathered}
$$

Objective function (5) minimizes the total weighted travel distance of all customers. Constraints (6) assign each customer $i$ to a single service facility. Constraints (7) guarantee that the demand of a customer is allocated to a service facility only if it has been located. Constraint (8) sets the number of service facilities to be installed. Constraints (9) define the domain of the variables. Clearly, in the PMP, the demand is assigned to a facility based only on the travel distance.

4.3. The Simulation Method. Real-world decision-making is usually affected by uncertainties, so an agent-based simulation model was built with AnyLogic to evaluate the network performance under input stochasticity. AnyLogic is one of the most powerful simulation packages, and by using multiple methods including system dynamics and agentbased and discrete-event modeling, it can be used to simulate complex systems with different features [61]. In our case study, we consider demand stochasticity in performance evaluation. The customer demands for postal service are generated in the real-world GIS with random locations, random time, and random demand. This will affect the objective values of the optimization model due to the stochasticity in parameters $d_{i}, c_{i j}$, and $N_{i}$. The movement of individual customers or agents is governed by the demand allocation strategy, which can be to go to a specific service facility or to travel to the nearest one for service.

The simulation model is, by nature, a stochastic form of the optimization model. It can be written as a generic objective function $f\left(y_{j}, \xi\left(d_{i}, c_{i j}, N_{i}\right)\right)$ to calculate either coverage (to be maximized) or distance (to be minimized), where $y_{j}$ is the location decision and $\xi$ is the scenario tree. With given probability distributions for the stochastic parameters, an infinite number of decision trees can be generated. However, we focus on the model's behavior but not on the scenario generation procedures [56], so the output of the simulation model should be stable with different scenario trees [74], as shown in equation (10). Therefore, a Monte Carlo simulation method is used, which runs the AnyLogic model for $|S|$ times in order to test the stability of the analytical results:

$$
f\left(\hat{y}_{j}, \xi_{p}\left(d_{i}, c_{i j}, N_{i}\right)\right) \approx f\left(\hat{y}_{j}, \xi_{q}\left(d_{i}, c_{i j}, N_{i}\right)\right), \quad \forall p, q \in S .
$$

Real-world problems can be extremely large and may therefore lead to a significant computational challenge. In this regard, instead of performing a full-size simulation with a large number of agents and a long-time horizon, a reliable sampling method may be used to approximate the objective value of the real problem, as shown in equation (11), where the sampling problem $\gamma$ is much smaller than that of the real problem $\xi$ :

$$
f\left(\hat{y}_{j}, \gamma\left(d_{i}, c_{i j}, N_{i}\right)\right) \approx f\left(\hat{y}_{j}, \xi\left(d_{i}, c_{i j}, N_{i}\right)\right), \quad \forall p \in S .
$$

When a smaller size simulation is used to analyze the real problem, both the stability and the quality of the sampling procedures must be checked. This idea is based on the sample average approximation (SAA) [75]. For a location decision $\widehat{y}_{j}$ and a given sample size, stability is checked with $|S|$ repetitions, as shown in equation (12). Then, the simulation is run with a set of increased samples generated from the same probability distribution. The gap between the objective values obtained is used to measure the quality of the approximation. If the gap estimators satisfy the quality criteria, the given sample size can be used to approximate the result of the real problem, as shown in equation (13). Otherwise, an increased sample size or repetition is needed.

$$
\begin{array}{ll}
f\left(\hat{y}_{j}, \gamma_{p}\left(d_{i}, c_{i j}, N_{i}\right)\right) \approx f\left(\hat{y}_{j}, \gamma_{q}\left(d_{i}, c_{i j}, N_{i}\right)\right), & \forall p, q \in S, \\
f\left(\hat{y}_{j}, \gamma_{p}\left(d_{i}, c_{i j}, N_{i}\right)\right) \approx f\left(\hat{y}_{j}, \xi\left(d_{i}, c_{i j}, N_{i}\right)\right), & \forall p, q \in S .
\end{array}
$$

\section{Application and Results}

In this section, the data generation procedures for both optimization and simulation are first introduced. Then, discussions are given based on the numerical results.

5.1. Data Generation and Model Implementation. To implement the optimization models for the urban postal service network redesign problem in Narvik, input data were first generated based on the following assumptions:

(1) The urban area of Narvik is divided into 40 same-size customer segments (A1, A2,..., E8), as shown in Figure 4.

(2) The customer demands for postal service are directly proportional to the population size of each customer segment. 


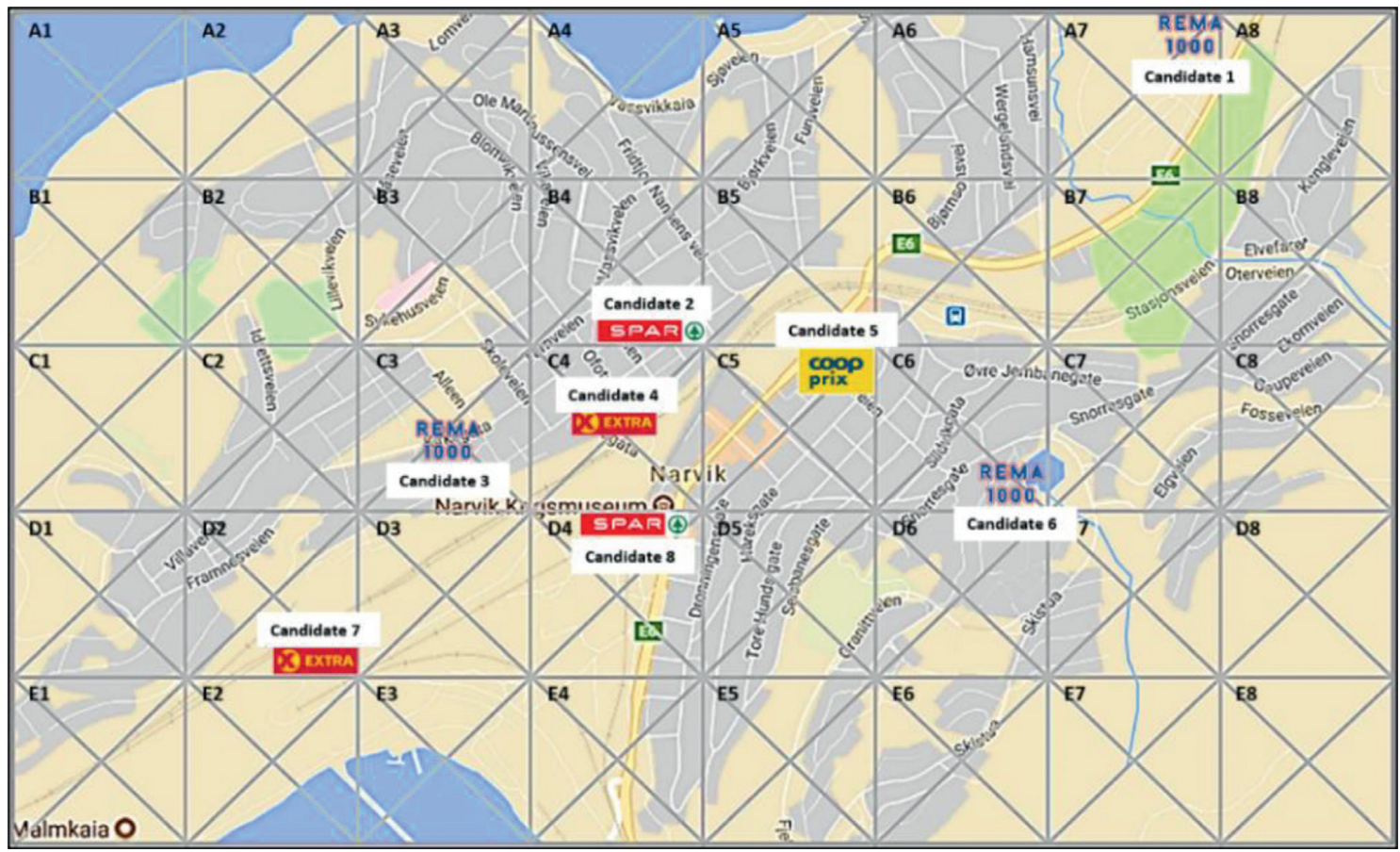

FIGURE 4: Customer demand aggregation and candidate locations for postal service counters.

(3) The population size is determined by the demographic distribution and by the portion of the residential area of each customer segment. Thus, the customer segments with very low coverage of the residential area, e.g., A1, are eliminated from the analysis. In total, 29 customer segments are included in the optimization.

(4) The weighted customer demands are aggregated into the center point of each customer segment.

The demand of each customer segment is first estimated. Customers need to go to the postal service counters to pick up their parcels and registered letters. Compared with the volume of registered letters delivered for individuals, the volume of parcel delivery for individuals is much higher. Therefore, the demand estimation is based mainly on parcel delivery with customer pickup at postal service counters. The total volume of parcel delivery in Norway is 59.9 million units [5], among which $60 \%$ is assumed to be parcel delivery for individuals with customer pickup at postal service counters, and the others are related to business demands and value-added home delivery. The total population in Norway is 5.296 million [76]. Hence, the average demand for parcel delivery with customer pickup per capita is approximately six to seven times a year, based on which the aggregated annual customer demand for postal service of each customer segment can be estimated by demand $=$ demand per capita $\times$ population.

The distance matrix between the center point of each customer segment and the candidate locations for postal service counters is calculated on Google Maps. The customer-preferred distance to the postal service counter is considered as the facility coverage radius, which was determined by interviews with randomly selected customers in Narvik. For a detailed discussion on the coverage radius of postal services in European countries, see [18]. In this study, the coverage radius of a postal service counter is set to $900 \mathrm{~m}$. Based on the distance matrix and coverage radius, the coverage matrix can be established as in Table 4. The optimization problems consist of 29 customer segments and eight candidate locations for postal service counters. Both MCLP and PMP were solved using LINGO 18.0. Since the sizes of the optimization problems are very small, the models can be solved within two seconds.

In the second stage, an agent-based simulation model was built in AnyLogic 8.4 to test different combinations of location plans and allocation strategies. Agent-based modeling $(\mathrm{ABM})$ is able to simulate the behaviors of individuals within a system as well as the interactions among them [77]. Hence, the characteristics of the urban postal system redesign problem can be better represented in the simulation. The simulation model was implemented under the following assumptions:

(1) The average number of people per family in Narvik is 2.13 [78], and the demand for postal services is seven times per person per year. In the simulation, one agent represents the customer demands from the same family. Considering uncertainty, customer demands were randomly generated from a continuous uniform distribution with an upper bound calculated by $c^{\text {upper }}=(1+\beta) c^{m}$ and a lower bound calculated as $c^{\text {lower }}=(1-\beta) c^{m}[79]$, where $c^{m}=14$ and $\beta=0.15$ were used.

(2) The map of Narvik was created with a real GIS (OSM classic), in which the customer demand points were 
TABLe 4: The coverage matrix.

\begin{tabular}{lc}
\hline Candidate & Demand coverage \\
\hline 1 & A6, B5, B6 \\
2 & A4, A5, B3, B4, B5, B6, C3, C4, C5, C6, D4, D5 \\
3 & A3, B2, B3, B4, C2, C3, C4, C5, D2, D4 \\
4 & A4, B4, B5, C3, C4, C5, C6, D4, D5, E4 \\
5 & A5, A6, B4, B5, B6, C4, C5, C6, C7, D5, D6 \\
6 & B6, C5, C6, C7, C8, D5, D6, D7, E6 \\
7 & $\mathrm{C} 3, \mathrm{D} 1, \mathrm{D} 2, \mathrm{D} 4$ \\
8 & $\mathrm{~B} 4, \mathrm{~B} 5, \mathrm{C} 3, \mathrm{C} 4, \mathrm{C} 5, \mathrm{C} 6, \mathrm{D} 4, \mathrm{D} 5, \mathrm{D} 6, \mathrm{E} 4$ \\
\hline
\end{tabular}

TABle 5: Scenarios with different location and demand allocation strategies.

\begin{tabular}{|c|c|c|c|c|}
\hline \multirow{2}{*}{ Scenarios } & \multirow{2}{*}{ Number of facilities } & \multicolumn{2}{|c|}{ Facility locations } & \multirow{2}{*}{ Allocation plan } \\
\hline & & Location plan & Candidate & \\
\hline S1 & 1 & Original & 5 & \\
\hline S2 & 1 & Optimal & 4 & \\
\hline S3 & 2 & Current & 2,7 & Current allocation plan \\
\hline S4 & 2 & Current & 2,7 & Optimal allocation plan \\
\hline S5 & 2 & Optimal & 3,5 & Current allocation plan \\
\hline S6 & 2 & Optimal & 3,5 & Optimal allocation plan \\
\hline
\end{tabular}

TABLe 6: Performance evaluation of different scenarios.

\begin{tabular}{|c|c|c|c|c|}
\hline \multirow{2}{*}{ Scenarios } & \multirow{2}{*}{ Demand coverage (\%) } & \multicolumn{2}{|c|}{ Travel distance $(\mathrm{m})$} & \multirow{2}{*}{ Standard deviation $(\mathrm{m})$} \\
\hline & & Mean & Overall median & \\
\hline S1 & 32.9 & 1220.3 & 1176.5 & 525.8 \\
\hline S2 & 31.7 & 1221 & 1175.5 & 532.8 \\
\hline S3 & 25.2 & 1271 & 1301.1 & 505.9 \\
\hline S4 & 35.6 & 1219.3 & 1177.4 & 557.2 \\
\hline S5 & 37.1 & 1227 & 1165.7 & 609 \\
\hline S6 & 49.2 & 945.5 & 918.3 & 417.5 \\
\hline
\end{tabular}

randomly generated over the residential area. The locations of the postal service counters in different scenarios were obtained from the optimization models. Besides, the travel distance between two points was calculated with real road information.

(3) Two demand allocation strategies were modeled. In the first one, customers are assigned to a postal service counter based only on their postcodes. For the other one, customers choose their nearest postal service counter.

(4) In the simulation, the sample size of demand points was set to 1,000 , and the virtual time for simulation was set to one year. For the stability check, 20 repetitions were performed for each scenario. The coefficient of variation (CV) was used for stability check, and the confidence level was set equal to $90 \%$.

(5) Due to the computational challenge, the full simulation could not be efficiently completed with the hardware configuration and software package used in the experiment. Therefore, instead of conducting a full-size simulation, four other scenarios with an increase in either the sample size $(2,000$ and 3,000$)$ or in the virtual time of simulation (three and five years) were tested for quality check. The expectation on the level of confidence in this step was set to $90 \%$.
All simulation models were run on a PC with the same configuration as that used in the first stage. The CPU time varies from 300 to 1,100 seconds with respect to different sample sizes and virtual time for simulation.

5.2. Results and Discussion. When the number of postal service counters installed is equal to one or two, both the MCLP and the PMP yield the same optimal location decisions. Considering several combinations of location-allocation plans, six scenarios were first compared, as shown in Table 5. When only one postal service counter is installed in scenarios S1 and S2, all customers are obviously assigned to the same location. Scenarios S3, S4, S5, and S6 are twofacility scenarios with different location and demand allocation strategies.

Table 6 shows the performance evaluation of the network accessibility with respect to two criteria: demand coverage and travel distance. When only one postal service counter is installed, the simulation result suggests that the difference between the original location and the optimal location is small, and the accessibility cannot be noticeably improved through location optimization. Next, the accessibility of the current two locations of the postal service counters and the current postcode-based demand allocation strategy is evaluated in S3. The performance of both 


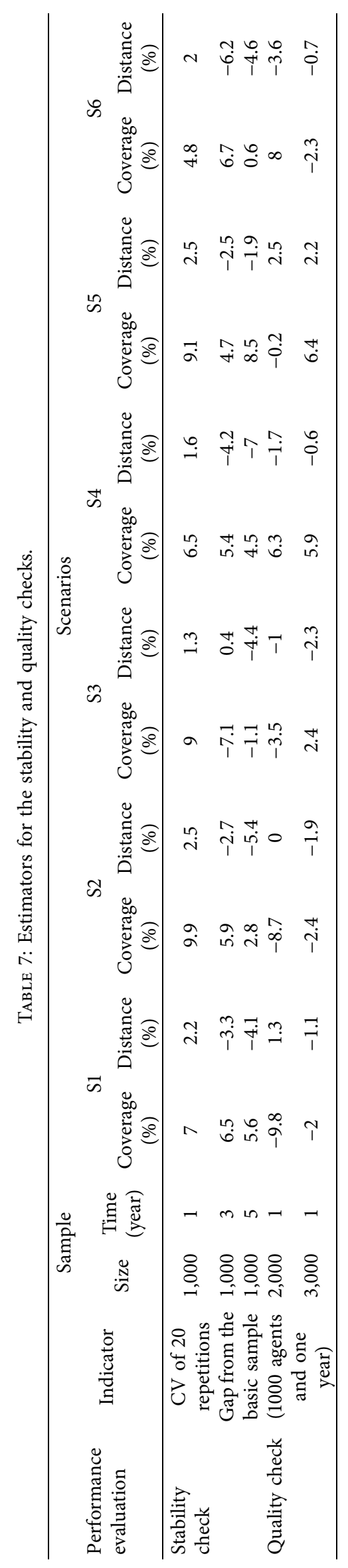


TABLE 8: Optimal decisions of the MCLP and the PMP with an increased number of facilities to be installed.

\begin{tabular}{lcc}
\hline Number of facilities $(p)$ & Optimal locations & $p$-median location problem \\
\hline 1 & Candidate 4 & Candidate 4 \\
2 & Candidates 3 and 5 & Candidates 3 and 5 \\
3 & Candidates 1,4 , and 6 & Candidates 3 , 4, and 6 \\
4 & Candidates $1,2,6$, and 7 & Candidates 1,3 , 4 , and 6 \\
5 & Candidates $1,2,3,6$, and 7 & Candidates $1,3,4,5$, and 6 \\
6 & Candidates $1,2,3,6,7$, and 8 & Candidates $1,3,4,5,6$, and 7 \\
7 & Candidates $1,2,3,4,6,7$, and 8 & Candidates $1,3,4,5,6,7$, and 8 \\
8 & Candidates $1,2,3,4,5,6,7$, and 8 & Candidates $1,2,3,4,5,6,7$, and 8 \\
\hline
\end{tabular}

demand coverage and travel distance is reduced compared with the one-facility scenarios, which shows that installing a larger number of facilities may not improve the network accessibility if an improper demand allocation strategy is implemented. When either the allocation strategy (S4) or the location decision (S5) is optimized, the result suggests that an improvement in the performance indictors may be achieved. Finally, when both the optimal location decision and the distance-based allocation strategy are implemented in S6, the accessibility of the urban postal service network can be drastically improved. Compared with the current plant, the demand coverage increases by $95.2 \%$, and the average travel distance reduces by $25.6 \%$. This result illustrates that the accessibility of a service network is determined not only by the number of facilities installed but also by the facility locations and by the demand allocation strategies implemented.

Due to the stochastic nature of the problem, the effective use of the simulation results depends on the stability and quality of the sample with respect to the real problem. Table 7 shows the stability and quality checks. First, the CV $(=\sigma / \mu)$ of the 20 repetitions with sample size 1,000 is used for the stability check, which ranges from $1.3 \%$ to $9.9 \%$. The average value of 20 repetitions is used as the benchmark. With an increase in either sample size or virtual time for simulation, the estimators for the quality check are calculated and normalized to the same scale of the benchmark scenario. The absolute values of these estimators range from 0 to $9.8 \%$. The evaluation result shows that both stability and quality fulfill the requirement of the level of confidence. In addition, we observe that the absolute values of the gap estimators for demand coverage are larger than those of the travel distance in most cases. This can be explained by the fact that the distance between customer locations and postal service counters is the only indicator for calculating the demand coverage, which may be significantly affected by the randomness related to the generation of customer locations and hence result in a high variation. However, on the contrary, the aggregation of the uncertainty related to both customer demand and geographical location in the calculation of total travel distance in the PMP may lead to a more stable result.

The sensitivity of the system performance with respect to the number of postal service counters opened is of interest. Table 8 shows the location decisions obtained by the MCLP and the PMP with an increase in the number of facilities opened. In the sensitivity analysis, only the optimal distancebased demand allocation strategy is considered. When $p=3$, $4,5,6$, and 7, the MCLP and the PMP yield different location decisions. Table 9 shows the gap estimators for the stability and the quality checks, which exhibit a pattern similar to that of the previous scenarios. Herein, the expected level of confidence for the quality check is relaxed to $87 \%$ in order to maintain the computational efficiency. In addition, it is noted that the change of virtual time may have more impact on the simulation results. Table 10 presents the computational performance of the simulation model in the sensitivity analysis. Compared with the change of the virtual time for simulation, the increase in the sample size has much more influence on the computational performance. Therefore, we have shown that the proposed method can effectively maintain its computational efficiency while providing, at the same time, a high level of confidence in the simulation results.

As shown in Figure 5, the result of the sensitivity analysis suggests that the accessibility of the urban postal service system in Narvik evaluated by both indicators can be improved when $p$ increases from one to three. However, accessibility may not be significantly improved by installing more than three postal service counters. This provides important information to the decision makers on the proper number of postal service counters to install under both accessibility and budget constraints. In addition, we observe that, in most cases, the optimal solution obtained by the PMP may outperform the one calculated by the MCLP in both demand coverage and travel distance. 


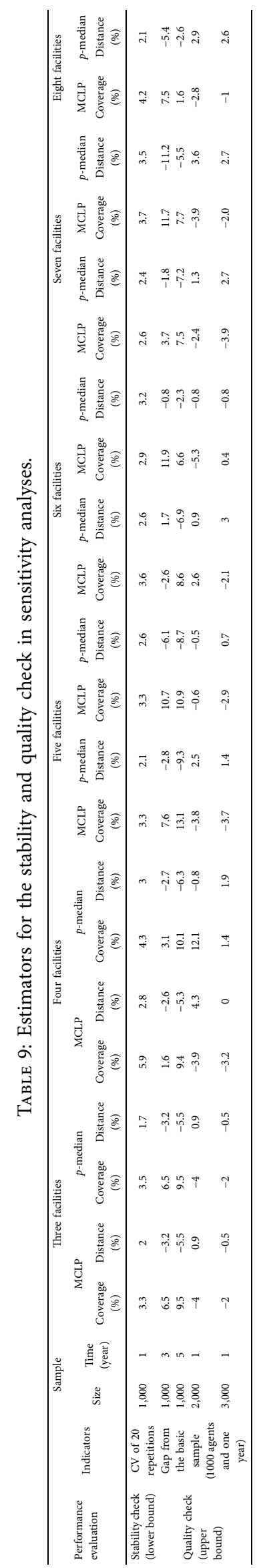




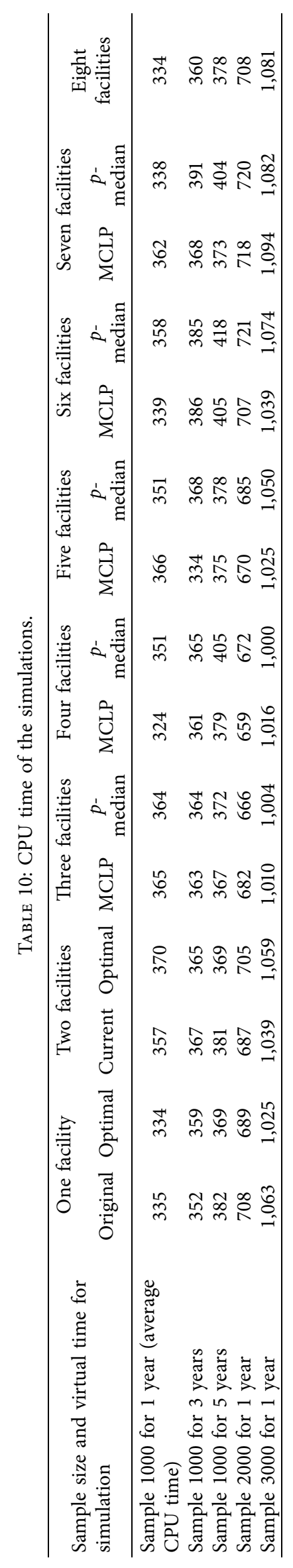




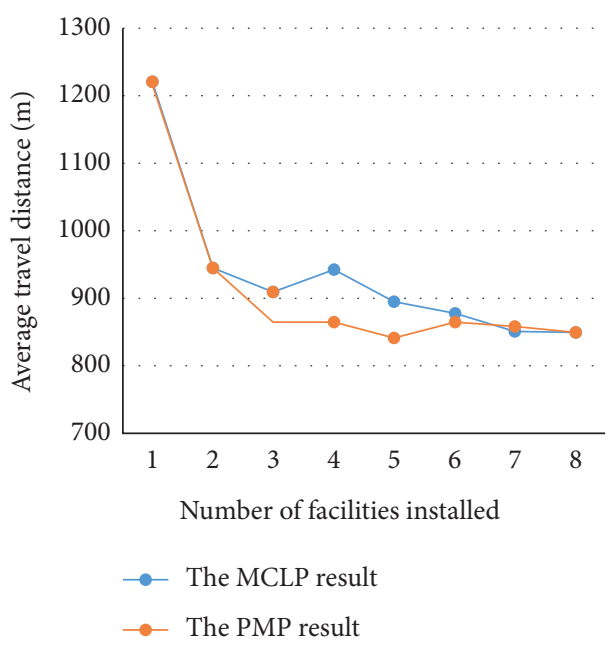

(a)

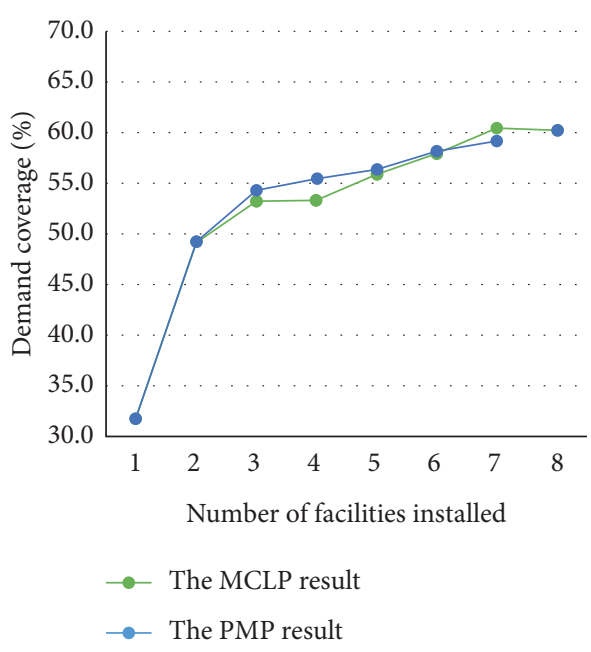

(b)

FIGURE 5: Result of the sensitivity analysis.

\section{Conclusions}

Facility location and demand allocation strategies are the most important factors affecting the accessibility of a service system. In this paper, we have proposed a two-stage approach for service system design and tested it with a realworld urban postal service system redesign problem in Norway. First, two mathematical models were used to determine the optimal number and locations of service facilities. Then, a simulation model was built to evaluate different location-allocation strategies. Considering the tradeoff between the quality of analytical results and the computational effort required, instead of performing fullsize simulations, sampling procedures based on the SAA were developed for solving large instances. The results of the case study in Narvik reveal that even if another postal service counter had been opened in the current system, the accessibility would have been reduced significantly due to the implementation of the postcode-based demand allocation strategy. When either the location or the demand allocation strategy is optimized, the impacts on the accessibility are relatively insignificant. However, when both optimal location decision and demand allocation strategy are implemented, the accessibility of the urban postal service network can be drastically improved. In addition, the experimental results also suggest that the proper number of facilities for maximizing the accessibility of the urban postal service system is three, beyond which the accessibility cannot be effectively improved with an increase in the number of postal service counters.

Among other insights, the research shows that both facility location and demand allocation strategies may play an important role in determining the accessibility of a service system. Besides, the accessibility may not be improved by increasing the number of facilities when the break-even point of the system performance is reached. From a methodological perspective, the proposed two-stage approach uses the strengths of both optimization and simulation. Mathematical models can effectively obtain optimal decisions with several simplifications. For instance, the aggregation of customer demands into a few points may work well in large systems with discrete customer locations such as investigating the performance of a rural healthcare network [28, 54]. In these systems, the distances between different customer locations are very large, and the demands of a city or a town can be properly aggregated at a higher level. However, in an urban area, this is more complicated due to the continuous distribution of residential areas from which customer demands are generated randomly in terms of time, location, and demand. In this case, aggregating customer demands into a large number of discrete points and taking into account the stochasticity may improve the accuracy of the analytical results, but, on the contrary, the modeling complexity and the computational requirements will be increased dramatically. In this regard, the simulation model can be effectively and efficiently used as a performance evaluation tool of the optimal location decisions and to analyze the system behavior under a more realistic environment with minimal assumptions and simplifications. Even though the proposed method is based on a specific problem, it can be applied to a wide range of service network planning problems, especially in urban areas.

Future work may be conducted to improve the current research in the following aspects:

(i) To better reflect the real-world situation, customers' trips for postal service may be categorized in more detail, and multitype tours with different purposes, origins, and destinations may be modeled in the simulation.

(ii) The system performance may further be investigated and compared with more criteria, e.g., equity of service [54] and facility utilization.

(iii) The current research only considers accessibility. However, when strategic alliance decisions with retailer stores are to be made, other influencing 
factors, i.e., the possible demand increase at shops due to combined customer trips, employee's satisfaction with increased demand, and multiple tasks, may also be considered in order to have a holistic analysis.

\section{Data Availability}

The data used in the experiment are available from the corresponding author upon request.

\section{Disclosure}

This paper is an extended and significantly modified version of "A Comparison of Two Location Models in Optimizing the Decision-Making on the Relocation Problem of Post Offices at Narvik, Norway" published at the IEEE International Conference on Industrial Engineering and Engineering Management, 2018, Bangkok, Thailand [80].

\section{Conflicts of Interest}

The authors declare that they have no conflicts of interest.

\section{References}

[1] G. Bruno, M. Cavola, A. Diglio, G. Laporte, and C. Piccolo, "Reorganizing postal collection operations in urban areas as a result of declining mail volumes-a case study in Bologna," Journal of the Operational Research Society, pp. 1-16, 2020.

[2] M. Šelmić, M. Nikolić, and A. Čupić, "Postboxes quantitative optimization model," Sustainability, vol. 12, no. 5, p. 1945, 2020.

[3] P. Norge, "Posten Norge: because people change Posten Norge much also change," 2013, https://nsd.no/polsys/data/ filer/aarsmeldinger/AE_2012_8608.pdf.

[4] S. Bellman, G. Lohse, and E. J. Johnson, "Predictors of online buying behavior," Communications of the ACM, vol. 42, pp. 32-48, 2009.

[5] P. Norge, "Posten Norge: annual and sustainability report 2019," 2019, https://www.postennorge.no/en/reportarchive/_/attachment/inline/2371ea4b-000c-4c2f-a4f86f0004f1c12c:becff691661c9172f74c3677f9c327921c8845d4/ Annual\%20and\%20sustainability\%20report\%202019_Posten \%20Norway\%5B1\%5D.pdf.

[6] J. Confraria, F. Silva, F. Pereira, and A. Franco, "Postal users' needs regarding accessibility to the postal network," in The Contribution of the Postal and Delivery Sector, Topics in Regulatory Economics and Policy, P. Parcu, T. Brennan, and V. Glass, Eds., Springer, Cham, Switzerland, pp. 187-205, 2018.

[7] S. H. Owen and M. S. Daskin, "Strategic facility location: a review," European Journal of Operational Research, vol. 111, no. 3, pp. 423-447, 1998.

[8] A. Klose and A. Drexl, "Facility location models for distribution system design," European Journal of Operational Research, vol. 162, no. 1, pp. 4-29, 2005.

[9] J. Yang and H. Lee, "An AHP decision model for facility location selection," Facilities, vol. 15, no. 9-10, pp. 241-254, 1997.

[10] B. Sennaroglu and G. Varlik Celebi, "A military airport location selection by AHP integrated PROMETHEE and
VIKOR methods," Transportation Research Part D: Transport and Environment, vol. 59, pp. 160-173, 2018.

[11] F. Zhang, D. M. Johnson, and J. W. Sutherland, "A GIS-based method for identifying the optimal location for a facility to convert forest biomass to biofuel," Biomass and Bioenergy, vol. 35, pp. 3951-3961, 2011.

[12] B. Bozkaya, S. Yanik, and S. Balcisoy, "A GIS-based optimization framework for competitive multi-facility locationrouting problem," Networks and Spatial Economics, vol. 10, no. 3, pp. 297-320, 2010.

[13] R. McCormack and G. Coates, "A simulation model to enable the optimization of ambulance fleet allocation and base station location for increased patient survival," European Journal of Operational Research, vol. 247, no. 1, pp. 294-309, 2015.

[14] R. Li and D. Tong, "Incorporating activity space and trip chaining into facility siting for accessibility maximization," Socio-Economic Planning Sciences, vol. 60, pp. 1-14, 2017

[15] S. Davari, M. H. Fazel Zarandi, and A. Hemmati, "Maximal covering location problem (MCLP) with fuzzy travel times," Expert Systems with Applications, vol. 38, no. 12, pp. 1453514541, 2011.

[16] S. Y. He, Y.-H. Kuo, and D. Wu, "Incorporating institutional and spatial factors in the selection of the optimal locations of public electric vehicle charging facilities: a case study of Beijing, China," Transportation Research Part C: Emerging Technologies, vol. 67, pp. 131-148, 2016.

[17] M. Blagojević, P. Ralević, and D. Šarac, "An integrated approach to analysing the cost efficiency of postal networks," Utilities Policy, vol. 62, Article ID 101002, 2020.

[18] D. Šarac, M. Kopić, K. Mostarac, M. Kujačić, and B. Jovanović, "Application of set covering location problem for organizing the public postal network," PROMET-Traffic\&Transportation, vol. 28 , no. 4 , pp. 403-413, 2016.

[19] G. Laporte, S. Nickel, and F. Saldanha da Gama, Location Science, Springer, Cham, Switzerland, 2nd edition, 2019.

[20] R. Church and C. ReVelle, "The maximal covering location problem," Papers of the Regional Science Association, vol. 32, no. 1, pp. 101-118, 1974.

[21] C. S. ReVelle and H. A. Eiselt, "Location analysis: a synthesis and survey," European Journal of Operational Research, vol. 165, no. 1, pp. 1-19, 2005.

[22] S. Atta, P. R. Sinha Mahapatra, and A. Mukhopadhyay, "Solving maximal covering location problem using genetic algorithm with local refinement," Soft Computing, vol. 22, no. 12, pp. 3891-3906, 2018.

[23] S. García and A. Marín, "Covering location problems," in Location Science, G. Laporte, S. Nickel, and F. Saldanha da Gama, Eds., pp. 99-119, Springer, Cham, Switzerland, Second Edition, 2019.

[24] T. H. Seyhan, L. V. Snyder, and Y. Zhang, "A new heuristic formulation for a competitive maximal covering location problem," Transportation Science, vol. 52, no. 5, pp. 1156-1173, 2018.

[25] O. Janković, S. Mišković, Z. Stanimirović, and R. Todosijević, "Novel formulations and VNS-based heuristics for single and multiple allocation $p$-hub maximal covering problems," $A n$ nals of Operations Research, vol. 259, pp. 191-216, 2017.

[26] M. A. Pereira, L. C. Coelho, L. A. N. Lorena, and L. C. De Souza, "A hybrid method for the probabilistic maximal covering location-allocation problem," Computers \& Operations Research, vol. 57, pp. 51-59, 2015.

[27] J.-F. Cordeau, F. Furini, and I. Ljubić, "Benders decomposition for very large scale partial set covering and maximal 
covering location problems," European Journal of Operational Research, vol. 275, no. 3, pp. 882-896, 2019.

[28] L. Murawski and R. L. Church, "Improving accessibility to rural health services: the maximal covering network improvement problem," Socio-Economic Planning Sciences, vol. 43, no. 2, pp. 102-110, 2009.

[29] V. Verter and S. D. Lapierre, "Location of preventive health care facilities," Annals of Operations Research, vol. 110, no. 1-4, pp. 123-132, 2002.

[30] P. Sorensen and R. Church, "Integrating expected coverage and local reliability for emergency medical services location problems," Socio-Economic Planning Sciences, vol. 44, no. 1, pp. 8-18, 2010.

[31] I. Frade and A. Ribeiro, "Bike-sharing stations: a maximal covering location approach," Transportation Research Part A: Policy and Practice, vol. 82, pp. 216-227, 2015.

[32] S. H. R. Pasandideh, S. T. A. Niaki, and M. Sheikhi, "A biobjective hub maximal covering location problem considering time-dependent reliability and the second type of coverage," International Journal of Management Science and Engineering Management, vol. 11, no. 4, pp. 195-202, 2016.

[33] X. Li, M. Ramshani, and Y. Huang, "Cooperative maximal covering models for humanitarian relief chain management," Computers \& Industrial Engineering, vol. 119, pp. 301-308, 2018.

[34] N. R. Paul, B. J. Lunday, and S. G. Nurre, "A multiobjective, maximal conditional covering location problem applied to the relocation of hierarchical emergency response facilities," Omega, vol. 66, pp. 147-158, 2017.

[35] S. L. Hakimi, "Optimum locations of switching centers and the absolute centers and medians of a graph," Operations Research, vol. 12, no. 3, pp. 450-459, 1964.

[36] S. L. Hakimi, "Optimum distribution of switching centers in a communication network and some related graph theoretic problems," Operations Research, vol. 13, no. 3, pp. 462-475, 1965.

[37] A. Marín and M. Pelegrín, "p-median problems," in Location Science, G. Laporte, S. Nickel, and F. Saldanha da Gama, Eds., pp. 20-50, Springer, Cham, Switzerland, 2nd edition, 2019.

[38] L. A. N. Lorena and E. L. F. Senne, "Local search heuristics for capacitated p-median problems," Networks and Spatial Economics, vol. 3, no. 4, pp. 407-419, 2003.

[39] I. M. Branco and J. D. Coelho, "The Hamiltonian p-median problem," European Journal of Operational Research, vol. 47, no. 1, pp. 86-95, 1990.

[40] J. F. Campbell, "Hub location and the $p$-hub median problem," Operations Research, vol. 44, no. 6, pp. 923-935, 1996.

[41] E. Alekseeva, N. Kochetova, Y. Kochetov, and A. Plyasunov, "A hybrid memetic algorithm for the competitive $p$-median problem," IFAC Proceedings Volumes, vol. 42, no. 4, pp. 1533-1537, 2009.

[42] N. Mladenović, J. Brimberg, P. Hansen, and J. A. MorenoPérez, "The $p$-median problem: a survey of metaheuristic approaches," European Journal of Operational Research, vol. 179, pp. 927-939, 2007.

[43] R. L. Church and S. Wang, "Solving the $p$-median problem on regular and lattice networks," Computers \& Operations Research, vol. 123, p. 105057, 2020.

[44] J. Q. Hale, E. Zhou, and J. Peng, “A Lagrangian search method for the P-median problem," Journal of Global Optimization, vol. 69, no. 1, pp. 137-156, 2017.

[45] G. Erdoğan, G. Laporte, and A. M. Rodríguez Chía, "Exact and heuristic algorithms for the Hamiltonian $p$-median problem,"
European Journal of Operational Research, vol. 253, pp. 280-289, 2016.

[46] T. Bektaş, L. E. Gouveia, and D. Santos, "Revisiting the Hamiltonian $p$-median problem: a new formulation on directed graphs and a branch-and-cut algorithm," European Journal of Operational Research, vol. 276, pp. 40-64, 2019.

[47] F. Stefanello, O. C. B. de Araújo, and F. M. Müller, "Matheuristics for the capacitated $p$-median problem," International Transactions in Operational Research, vol. 22, no. 1, pp. 149-167, 2015.

[48] Z. Drezner and S. Salhi, "Incorporating neighborhood reduction for the solution of the planar $p$-median problem," Annals of Operations Research, vol. 258, no. 2, pp. 639-654, 2017.

[49] J. M. Colmenar, P. Greistorfer, R. Martí, and A. Duarte, "Advanced greedy randomized adaptive search procedure for the obnoxious $p$-median problem," European Journal of Operational Research, vol. 252, no. 2, pp. 432-442, 2016.

[50] B. F. De Azevedo and N. D. Pizzolato, Satoru Ochi, "Proposal for analysis of location of popular residential using the $p$ median," in Smart and Digital Cities, V. Nazário Coelho, I. Machado Coelho, and T. Aparecida de Oliveira, Eds., Springer, Cham, Switzerland, pp. 65-77, 2019.

[51] N. Adler, E. T. Njoya, and N. Volta, "The multi-airline $p$-hub median problem applied to the African aviation market," Transportation Research Part A: Policy and Practice, vol. 107, pp. 187-202, 2018.

[52] A. P. Wheeler, "Creating optimal patrol areas using the $p$ median model," Policing: An International Journal, vol. 42, no. 3, pp. 318-333, 2019.

[53] C. Cintrano, F. Chicano, T. Stützle, and E. Alba, "Studying solutions of the $p$-median problem for the location of public bike stations," Lecture Notes in Computer Science, Springer, Berlin, Germany, pp. 198-208, Article ID 11160, 2018.

[54] M. L. Burkey, J. Bhadury, and H. A. Eiselt, "A location-based comparison of health care services in four U.S. states with efficiency and equity," Socio-Economic Planning Sciences, vol. 46, no. 2, pp. 157-163, 2012.

[55] H. Yu, W. D. Solvang, and J. G. Yang, "Improving accessibility and efficiency of service facility through location-based approach: a case study at Narvik university college," Advanced Materials Research, vol. 1039, pp. 593-602, 2014.

[56] A. J. King and S. W. Wallace, Modeling with Stochastic Programming, Springer-Verlag, New York, NY, USA, 2012.

[57] J. Li, M. González, and Y. Zhu, "A hybrid simulation optimization method for production planning of dedicated remanufacturing," International Journal of Production Economics, vol. 117, no. 2, pp. 286-301, 2009.

[58] S. Lidberg, T. Aslam, L. Pehrsson, and A. H. C. Ng, "Optimizing real-world factory flows using aggregated discrete event simulation modelling," Flexible Services and Manufacturing Journal, vol. 32, no. 4, pp. 888-912, 2019.

[59] X. Sun, H. Yu, and W. D. Solvang, "Solving the location problem of printers in a university campus using p-median location model and AnyLogic simulation," in Proceedings of the International Workshop of Advanced Manufacturing and Automation, pp. 577-584, Springer, Plymouth, UK, November 2019.

[60] M. Hrušovský, E. Demir, W. Jammernegg, and T. Van Woensel, "Hybrid simulation and optimization approach for green intermodal transportation problem with travel time uncertainty," Flexible Services and Manufacturing Journal, vol. 30, pp. 486-516, 2018. 
[61] Y. Yang, J. Li, and Q. Zhao, "Study on passenger flow simulation in urban subway station based on anylogic," Journal of Software, vol. 9, pp. 140-146, 2014.

[62] D. Mourtzis, M. Doukas, and D. Bernidaki, "Simulation in manufacturing: review and challenges," Procedia CIRP, vol. 25, pp. 213-229, 2014.

[63] S. Lupin, K. N. Z. Lin, H. Tun, A. M. Thike, and H. H. Linn, "Data structure for GIS based firefighting stations simulations," in Proceeding of the IEEE Conference of Russian Young Researchers in Electrical and Electronic Engineering (EIConRus), pp. 1545-1548, Moscow, Russia, January 2018.

[64] J. Rouzafzoon and P. Helo, "Developing service supply chains by using agent based simulation," Industrial Management \& Data Systems, vol. 116, no. 2, pp. 255-270, 2016.

[65] P. Helo, J. Rouzafzoon, and A. Gunasekaran, "Service supply chain design by using agent-based simulation," in Practices and Tools for Servitization, M. Kohtamäki, T. Baines, R. Rabetino, and A. Bigdeli, Eds., Palgrave Macmillan, Cham, Switzerland, pp. 387-403, 2018.

[66] X. Li, R. Kizito, and T. I. Paula, "An agent-based simulation framework for supply chain disruptions and facility fortification," in Proceedings of the 2018 Winter Simulation Conference, pp. 821-832, Gothenburg,, December 2018.

[67] S. Kim, S. Kim, and J. R. Kiniry, "Two-phase simulation-based location-allocation optimization of biomass storage distribution," Simulation Modelling Practice and Theory, vol. 86, pp. 155-168, 2018.

[68] V. Elia, M. G. Gnoni, and F. Tornese, "Designing a sustainable dynamic collection service for WEEE: an economic and environmental analysis through simulation," Waste Management \& Research: The Journal for a Sustainable Circular Economy, vol. 37, no. 4, pp. 402-411, 2019.

[69] V. Marianov and C. ReVelle, "The queueing maximal availability location problem: a model for the siting of emergency vehicles," European Journal of Operational Research, vol. 93, no. 1, pp. 110-120, 1996.

[70] R. Swamy, J. E. Kang, R. Batta, and Y. Chung, "Hurricane evacuation planning using public transportation," SocioEconomic Planning Sciences, vol. 59, pp. 43-55, 2017.

[71] E. Tekin and I. Sabuncuoglu, "Simulation optimization: a comprehensive review on theory and applications," IIE Transactions, vol. 36, no. 11, pp. 1067-1081, 2004.

[72] S. Ahmed, "Two-stage stochastic integer programming: a brief introduction," in Wiley Encyclopedia of Operations Research and Management Science, J. J. Cochran, L. A. Cox, P. Keskinocak et al., Eds., Wiley, Hoboken, NJ, USA, 2010.

[73] S. Attia, E. Gratia, A. De Herde, and J. L. M. Hensen, "Simulation-based decision support tool for early stages of zero-energy building design," Energy and Buildings, vol. 49, pp. 2-15, 2012.

[74] H. Yu, X. Sun, W. D. Solvang, G. Laporte, and C. K. M. Lee, “A stochastic network design problem for hazardous waste management," Journal of Cleaner Production, vol. 277, Article ID 123566, 2020.

[75] A. J. Kleywegt, A. Shapiro, and T. Homem-De-Mello, "The sample average approximation method for stochastic discrete optimization," SIAM Journal on Optimization, vol. 12, no. 2, pp. 479-502, 2002.

[76] SSB, "Key figures for the population," 2019, https://www.ssb. no/en/befolkning/nokkeltall/population.

[77] B. Wang, S. Brême, and Y. B. Moon, "Hybrid modeling and simulation for complementing lifecycle assessment," Computers \& Industrial Engineering, vol. 69, pp. 77-88, 2014.
[78] Municipality of Narvik, “Demography," 2016, https://ugeo. urbistat.com/AdminStat/en/no/demografia/dati-sintesi/ narvik/20485108/4.

[79] M. S. Pishvaee and S. A. Torabi, "A possibilistic programming approach for closed-loop supply chain network design under uncertainty," Fuzzy Sets and Systems, vol. 161, no. 20, pp. 2668-2683, 2010.

[80] H. Yu and W. D. Solvang, "A comparison of two location models in optimizing the decision-making on the relocation problem of post offices at Narvik, Norway," in Proceedings of the IEEE International Conference on Industrial Engineering and Engineering Management (IEEM), pp. 814-818, Bangkok, Thailand, December 2018. 